\title{
Initial experience in the extraction of chronically implanted pacemaker leads using the Excimer laser sheath
}

T Levy, S Walker, V Paul

\begin{abstract}
Objective-To assess the safety and efficiency of the Excimer laser sheath in extracting chronically implanted pacemaker leads.

Patients-Eight patients were studied (one female, mean age 62 years, range 34 to 77 ) with 17 pacemaker leads (five atrial, 10 ventricular, two implantable defibrillator). The mean implantation time was 65 months (range 23 to 188). The indications for lead extraction were chronic infection (7), superior vena cava obstruction (4), lead malfunction (4), and pain (2).

Methods-A prospective analysis of the use of the Excimer laser sheath in extracting chronically implanted pacemaker leads. Laser sheath extraction was undertaken if conventional extraction techniques with simple traction or traction with a locking stylet had failed. If laser sheath extraction was unsuccessful, basket retrieval of the lead from the groin was performed.

Results-Complete lead removal was achieved in 16 leads (94\%). In one case the electrode tip was left behind without complication. Extraction was achieved with the laser sheath alone in 16 leads. Basket retrieval was required in one case after laser failure. There were no complications.

Conclusions-The Excimer laser sheath appears to be an effective and safe technique for extracting chronically implanted pacemaker leads. It can be used in combination with the currently available techniques for successful lead extraction. (Heart 1999;82:101-104)
\end{abstract}

Keywords: Excimer laser sheath; lead extraction; pacing

Chronically implanted pacemaker leads develop numerous fibrous adhesions between the lead itself and the venous endothelium. These adhesions can prevent the successful removal of malfunctioning pacemaker leads. Malfunctioning leads are often left in situ with the potential long term complications of infection, subclavian vein thrombosis, and tricuspid valve dysfunction.

We describe our initial experience with the safety and efficacy of the Excimer laser sheath in the removal of 17 chronically implanted pacemaker leads in eight patients. The Excimer laser is a xenon chloride laser with an output of
$308 \mathrm{~nm}$ (Spectranetics Inc, Nieuwegein, The Netherlands). It is a cool cutting laser with an absorption depth of $0.06 \mathrm{~mm}$, cutting tissue only in contact with its tip. Only proteins and fats will absorb the laser's energy. The laser will cut through fibrous adhesions surrounding the pacemaker lead without damaging the venous endothelium or lead insulation and tines. The flexible laser sheath is placed over the pacemaker lead and transmits the laser energy to the sheath tip by fibreoptic channels within its wall. The sheath is available in sizes $12 \mathrm{~F}$ to $16 \mathrm{~F}$.

We describe the technique and first use of the Excimer laser sheath in the United Kingdom.

\section{Methods}

STUDY SETTING AND POPULATION

From April 1997 until February 1998, 17 leads (five atrial, 10 ventricular, and two implantable cardioverter defibrillator) from eight patients (one female, seven male; mean age 62 years, range 34 to 77 ) were extracted using the laser sheath. The mean implantation time of the leads was 65 months (range 23 to 188 months). One patient required removal of a single lead, while seven patients required multiple lead extraction (two leads in six patients and four leads in one patient). Clinical details are shown in table 1 .

Three patients were referred to our hospital following failure to extract leads with simple traction elsewhere (cases 3, 4, and 6). In the remaining five patients traction with a locking stylet had failed at our institution before the laser sheath was used.

Extraction procedures were performed in the cardiac catheterisation laboratory with continuous electrocardiographic monitoring. Local anaesthesia was used with benzodiazepine sedation or diamorphine if required. General anaesthesia was only used for patient expressed preference. Surgical cover was available.

LASER SHEATH EXTRACTION TECHNIQUE

The pacemaker generator is explanted and the electrodes mobilised. Any silk ties or pacemaker sleeves are removed, with the lead cut distal to the connector pins. The subsequent technique is similar to the Cook's non-laser extraction method. Briefly, the insulation layers are serially peeled back to allow suture to the inner and outer coils. A locking stylet is passed as far as possible into the tip of the pacemaker electrode and secured. The integrity is checked by gentle traction. Vicryl sutures are placed 
Table 1 Background information on the patients and leads extracted by the laser sheath and results for lasing time, fluoroscopy time, and total procedure time

\begin{tabular}{|c|c|c|c|c|c|c|c|}
\hline Case & Sex/age & Reason for explant & Lead type & $\begin{array}{c}\text { Time lead } \\
\text { implanted } \\
\text { (months) }\end{array}$ & $\begin{array}{l}\text { Lasing time } \\
(s)\end{array}$ & $\begin{array}{l}\text { Fluoroscopy time } \\
\text { (min) }\end{array}$ & $\begin{array}{l}\text { Lab time } \\
\text { (min) }\end{array}$ \\
\hline \multirow[t]{2}{*}{1} & $\mathrm{M} / 74$ & Lead malfunction & A & 56 & 135 & 27 & 210 \\
\hline & & & V & 56 & 148 & & \\
\hline \multirow[t]{2}{*}{2} & $\mathrm{M} / 37$ & SVC obstruction & $\mathrm{V}^{\star}$ & 42 & 139 & 60 & 170 \\
\hline & & & V & 188 & 250 & & \\
\hline \multirow[t]{2}{*}{3} & $\mathrm{M} / 60$ & SC obstruction & A & 99 & 713 & 44 & 160 \\
\hline & & Lead malfunction & $\mathrm{V}$ & 99 & 138 & & \\
\hline \multirow[t]{2}{*}{4} & $\mathrm{M} / 66$ & Chronic draining sinus & A & 36 & 577 & 55 & 190 \\
\hline & & & V & 36 & 319 & & \\
\hline \multirow[t]{2}{*}{5} & $\mathrm{M} / 71$ & Lead malfunction & ICD SVC & 24 & 134 & 34 & 205 \\
\hline & & & ICD V & 24 & 237 & & \\
\hline \multirow[t]{4}{*}{6} & $\mathrm{~F} / 76$ & Chronic draining sinus & A & 23 & 150 & 49 & 325 \\
\hline & & & $\mathrm{V}$ & 23 & 20 & & \\
\hline & & & $\mathrm{V}$ & 60 & 297 & & \\
\hline & & & $\mathrm{V}$ & 83 & 150 & & \\
\hline 7 & $\mathrm{M} / 77$ & Chronic draining sinus & $\mathrm{V}$ & 131 & 315 & 30 & 90 \\
\hline \multirow[t]{2}{*}{8} & $\mathrm{M} / 34$ & Pain & A & 60 & 90 & 22 & 180 \\
\hline & & & $\mathrm{V}$ & 60 & 185 & & \\
\hline
\end{tabular}

*Active fixation.

A, atrial lead; ICD, implantable cardioverter defibrillator lead; Lab, laboratory; SC, subclavian vein; SVC, superior vena cava; V, ventricular lead.

around both the inner and outer insulation layers to further secure the locking stylet. With active fixation leads rotation to unscrew the lead is performed before any traction is applied. Once the locking stylet is secure, attempts are made at this stage to remove the lead with traction; if unsuccessful the laser sheath is then used to cut through the adhesions.

Sizing of the laser sheath is necessary, with $12 \mathrm{~F}$ permitting removal of leads with an outer diameter of 7.1 F. Bigger sheaths (14-16 F) are required for larger pacing leads such as ICD leads. The laser sheath, surrounded by an outer sheath of polypropylene/Teflon, is passed over the lead assembly. The outer sheath helps to prevent buckling of the laser sheath and relieves friction from surrounding scar tissue. This allows longitudinal force to reach the laser tip. The outer sheath is normally $2-3 \mathrm{~cm}$ short of the laser tip, but it is possible to lase within the outer sheath. The laser sheath is then advanced with firm pressure while lasing, with counter traction supplied through the locking stylet. The technique is similar to that used with dilator sheaths, except that the laser takes the place of the inner sheath with less force required, the emphasis placed on cutting not pushing with the laser. The laser sheath often has to be completely advanced to the myocardial insertion point to free all adhesions.

Lasing along the ventricular wall may induce ventricular tachycardia, which will resolve without sequelae when the lasing stops.

Lasing should stop $1 \mathrm{~cm}$ from the distal end of the lead tip to prevent cutting the myocardium. If the lead cannot be removed with gentle traction on the locking stylet at this time, the outer sheath can be advanced to the myocardial wall and used for counter traction to free the lead tip. If implantation of a new lead is required the outer sheath can be left in place and the new lead passed down it. The outer sheath is then cut longitudinally with scissors, taking care not to damage the new lead. Haemostasis is achieved by simple pressure.
Patients are monitored overnight and discharged the following day, if otherwise well. If the leads are removed for pacemaker infection we recommend the use of a temporary pacemaker wire to cover a period of antibiotic use before a new system is implanted.

\section{Results}

Complete removal was achieved in 16 of 17 leads. In one lead (case 7) the electrode tip separated from the main lead and was left attached to the myocardium. The laser was successful in freeing all adhesions except in one lead (case 2), where it could make no further progress once the SVC had been reached. This was probably because of calcification of the fibrous tissue at this site. This lead was 15 years old and complete removal was achieved by the right femoral approach using basket retrieval. The second lead in this case was removed using the laser sheath. A $12 \mathrm{~F}$ laser was used in 15 leads and a $14 \mathrm{~F}$ in two leads (ICD leads).

Mean lasing time was 235 seconds per lead (range 20 to 713 seconds). Mean fluoroscopy time was 40 minutes/patient (range 22 to 60 minutes). This time includes the reimplantation of three new leads in two patients. The mean laboratory time (from when the patient enters the catheter laboratory to when they leave it) was 190 minutes (range 90 to 210 minutes). The mean time required to remove each lead was 90 minutes.

Implantation of new leads took place at the same time as extraction in cases 1 and 5 (table 1). In case 1 , both atrial and ventricular leads were replaced using the outer sheaths as an introducer, while in case 5, who had two ICD leads, only the right ventricular apical lead required replacement. Cases 2, 3, 6, and 8 were also pacemaker dependent. Case 2 with SVC obstruction had an abdominal pacemaker implanted before removal of the transvenous leads. Case 3, with subclavian vein obstruction and lead malfunction, had a new system implanted on the opposite side one month before extraction of the old system. Case 6, 
with chronic infection, had a temporary pacing wire for one week before a new system was implanted. Case 8 had an abdominal system implanted owing to intolerance of a pectoral system, again after a period of temporary pacemaker cover. Cases 4 and 7 were not pacing dependent and had new pacemaker systems implanted at later dates.

The procedure was completed and well tolerated using local anaesthetic in six cases. In case 6 (four leads removed) the procedure started under local anaesthesia but had to be completed under general anaesthesia because of back pain secondary to osteoarthritis. Case 8 requested a general anaesthetic from the onset. There were no complications related to the procedure, and patients were normally discharged within 24 hours unless they were required to stay in for other reasons.

\section{Discussion}

In our initial experience with the laser sheath we were able to completely extract 16 of 17 chronically implanted pacemaker leads without complication. There was one incomplete removal. In that case the electrode tip separated from the main lead and was left attached to the myocardium without harmful sequelae.

In our experience there were several other cases where the electrode tip began to separate from the main lead owing to unravelling and breakage of conductor coils. We feel this may be because the locking stylet is not at the electrode tip, although the stylet was advanced as far as possible in each case. We were able to remove these leads successfully by decreasing the traction applied through the locking stylet and advancing the laser sheath. There was also one lead in which complete extraction could not be achieved with the laser sheath alone. In this case basket retrieval from the right femoral vein was required. It is possible that primary extraction using basket retrieval might have failed in this case as a number of proximal adhesions had been divided with the laser sheath allowing subsequent successful basket retrieval. This case illustrates how a combination of complimentary techniques may be required to achieve successful lead removal.

One patient (case 3) had a new DDD system implanted before extraction on the opposite side. In this case successful removal of both old leads was achieved without causing any displacement or damage to the new leads.

The procedure is not quick, taking over three hours for each patient or about 90 minutes a lead. It is reasonable well tolerated, and in our opinion can be done under local anaesthetic.

Comparisons between transvenous techniques are available. The PLEXES trial (pacing lead explant with the excimer sheath) is a randomised trial comparing laser sheath extraction with the non-laser technique of locking stylet and dilator sheaths. The results have been published in abstract form ${ }^{1} ; 465$ patients requiring lead extractions have been randomised between the two methods. Laser was successful in $94 \%$ of leads, while non-laser in only $65 \%$. The unsuccessful attempts with non-laser crossed to laser sheath removal, which was successful in $88 \%$ of cases. Complications occurred in $1 \%$ of the laser group, and worryingly there was one death $(0.32 \%)$. Full publication is awaited to clarify the safety question.

Other publications are again in abstract form and are individual series. Lloyd et al reviewed their experience of 127 atrial lead extractions using mechanical and laser techniques. ${ }^{2}$ They reported that laser required less effort and time. The laser technique also required the shorter fluoroscopy time of 609 seconds, versus 970 seconds in the mechanical group. There was just one complication in the laser group compared with 12 with mechanical methods. Byrd reports similar findings. ${ }^{3} \mathrm{He}$ reviewed 10 years experience of extracting 1369 leads. In this large series all extraction techniques were used, with the mechanical methods causing lead breakage in $10-12 \%$ of cases. Lead breakage occurred with the laser method in only $0.7 \%$. His conclusion was that the laser sheath technique is more effective than mechanical methods for removing leads intact.

All of the above reports are from North America and involve large institutions that specialise in performing lead extractions. In Europe the situation is different and there is less experience with this device, although the first European experience has been published. ${ }^{4}$ This series consisted of 50 leads in 45 patients with success in 47 leads, and three lead parts left behind with no complications. The mean extraction time defined from the start of lasing to extraction was 10 minutes. This is certainly quicker than our experience, but the majority of the leads were single and had been implanted for a mean time of 47 months, compared with our 65 months. It was also noticed in that study that most fibrous tissue was present at the site of venous entry and the upper portion of the SVC. Although we also found adhesions in these areas, our experience is that the densest adhesions are found where two leads intertwine. These areas were difficult to lase and explain our longer procedure time.

Our results will be part of a larger registry data, PLESSE (pacing lead extraction surveillance study in Europe). This database is evaluating the use of the laser sheath and its complications in the European setting. Initial results of 113 leads extracted from 92 patients show complete extraction in $92 \%$, with just one complication of haemothorax and haemopericardium requiring no surgical intervention. All patients were well on hospital discharge after a mean stay of three days. ${ }^{5}$

In conclusion we have reported the first experience in the United Kingdom of the extraction of chronically implanted pacemaker leads with the Excimer laser sheath. It appears to be a safe and effective technique, but is relatively expensive, with each laser sheath currently costing approximately $£ 1000$. We would recommend using a staged approach. Initial extraction could be attempted locally using simple traction. If unsuccessful, referral to a regional centre with experience in lead extraction with surgical back up should follow. Our approach would then be to attempt extraction 
using the locking stylet, moving on to dilator sheaths if unsuccessful. The laser sheath should be reserved for the $30-40 \%$ of cases where these two techniques will fail.

It may be difficult in the future to justify lead extraction in centres that do not have laser as backup owing to its demonstrated superiority, especially where the indication for lead extraction is absolute. In these circumstances, when there can be considerable morbidity and mortality from retained leads, the cost of the laser sheath is justified. We believe the laser sheath is a useful technique currently to be reserved for problematic extractions. However, we feel its use will expand as further experience is gained and the cost decreases.

1 Byrd, B Wilkoff, C Love, et al. Clinical study of the laser sheath: result of the PLEXES trial [abstract]. PACE 1997;20:1053.

2 MA Lloyd, DL Hayes, MJ Osborn, et al. Telectronics Accufix 330-801 atrial J lead: extraction results utilising raditional and laser techniques [abstract]. PACE 1997;20: 1053.

3 CL Byrd. Lead extraction: ten-year experience [abstract]. PACE 1997;20:1110

$4 \mathrm{C}$ Kennergren. First European experience using examiner laser for the extraction of permanent pacemaker leads. PACE 1998;21:268-70.

5 C Kennergen, F Bracke, C Butter, et al. Excimer laser assisted extraction of permanent pacemaker leads: the Present European Multicentre Experience [abstract]. PACE 1998;21:817.

\section{IMAGES IN CARDIOLOGY}

\section{Apparent fracture of a pacemaker lead}

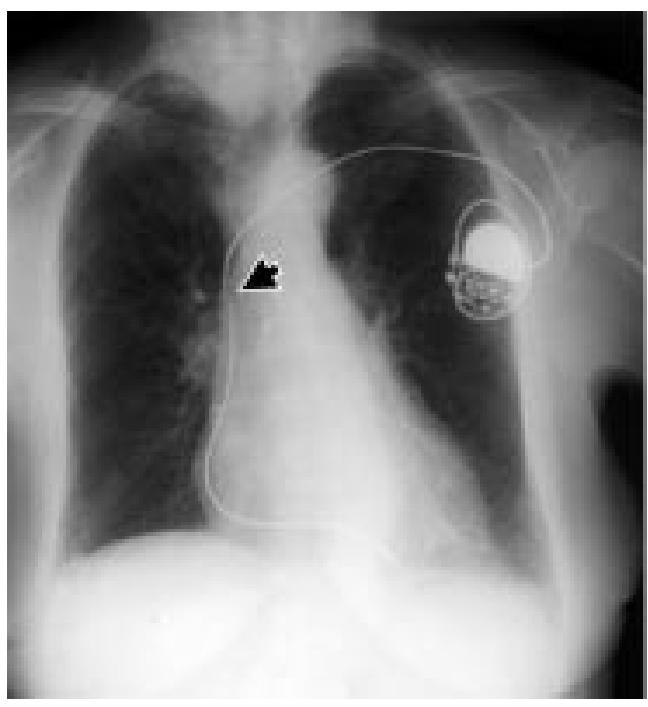

An 82 year old woman with symptomatic severe aortic stenosis underwent aortic valve replacement with a stentless porcine valve. This was complicated by complete heart block, which failed to resolve after six days. Because of atrial fibrillation at the time of implant, a single chamber (VVI-R) pacemaker was chosen. At implant, lead position and pacing parameters were satisfactory. At two month follow up pacing parameters were again satisfactory, but a chest radiograph using the advanced multiple beam equalisation radiography (AMBER) technique demonstrated lead fracture (left). A repeat standard chest radiograph showed an intact lead (right).

With the AMBER system the $x$ ray beam is passed through a modulator to divide it into 21 channels. Each channel has its own microproc-

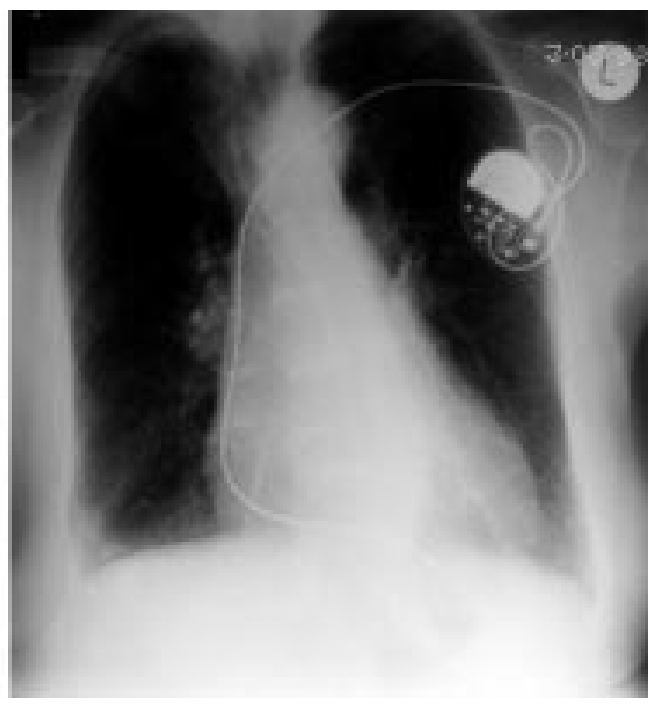

essor and detector, and can be adjusted to provide the correct film density. This compensates for attenuation differences in different parts of the chest. The patient's chest is scanned from bottom to top in 0.8 seconds. The apparent fracture can be explained by the longer exposure time of the AMBER. ${ }^{1}$ It should not be confused with pseudofracture, which is a well recognised radiographic pattern attributable to a very tight stay suture on the lead. ${ }^{2}$

D HEAVEN A ROCKALL R SUTTON

1 Fransson SG. Pacemaker wires. Difference in performance between AMBER and conventional chest radiography. Acta Radiol 1993:34:419-21.

2 Sutton R, Bourgeois I. The foundation of cardiac pacing. An illustrated practical guide, part 1. Mount Kisco, NY: Futura Publishing, 1991:286. 